\title{
Construção de um Vídeo Educativo sobre Detecção Precoce do Câncer de Mama
}

\author{
Elaboration of an Educational Video about Early Detection of Breast Cancer \\ Construcción de un Video Educativo sobre la Detección Precoz del Cáncer de \\ Mama
}

\author{
Camila Brasil Moreira ${ }^{1}$; Elizian Braga Rodrigues Bernardo ${ }^{2}$; Hellen Lívia Oliveira Catunda ${ }^{3}$; Priscila de Souza Aquino ${ }^{4}$; Míria Conceição Lavinas Santos ${ }^{5}$; \\ Ana Fátima Carvalho Fernandes ${ }^{6}$
}

\section{Resumo}

Introduçáo: As atividades de educação em saúde estimulam a prevenção de doenças, a promoção da saúde e o engajamento da população em assuntos relacionados à saúde e à qualidade de vida, através da construção do conhecimento. Objetivo: Descrever a construção de um vídeo educativo sobre o câncer de mama em uma oficina virtual. Método: Estudo do tipo de desenvolvimento de tecnologia educativa. A construção do material educativo ocorreu em junho de 2012, em quatro etapas: revisão narrativa da literatura, análise dos dados coletados, seleção dos dados e construção do material. Resultados: Obteve-se um vídeo educativo que abordava a definição do câncer de mama, as alteraçôes na mama sugestivas de câncer e os métodos de detecção da doença: mamografia, exame clínico das mamas, ultrassonografia mamária e autoexame das mamas. Conclusão: A enfermeira como profissional da saúde é responsável pela promoção da saúde da comunidade, devendo desenvolver avanços na enfermagem que assegurem o compromisso social, a luta e o combate às doenças, como a elaboração de materiais que auxiliem a educação em saúde. A oficina virtual como uma tecnologia educativa pode facilitar a construção do conhecimento de forma a promover saúde. Palavras-chave: Neoplasias de Mama-prevenção \& controle; Diagnóstico Precoce; Recursos Audiovisuais; Tecnologia Educacional; Comunicação em Saúde

\footnotetext{
${ }^{1}$ Acadêmica de Enfermagem da Universidade Federal do Ceará (UFC). Bolsista de Iniciação Científica PIBIC/CNPq. Fortaleza (CE), Brasil. E-mail: camilabrasil@alu.ufc.br. ${ }^{2}$ Acadêmica de Enfermagem da UFC. Bolsista do Programa de Educaçāo Tutorial - PET Enfermagem UFC. Fortaleza (CE), Brasil. E-mail: elizian_rodrigues@yahoo.com.br. ${ }^{3}$ Acadêmica de Enfermagem da UFC. Bolsista do Programa de Educaçăo Tutorial - PET Enfermagem UFC. Fortaleza (CE), Brasil. E-mail: hellen_enfermagem@yahoo.com.br

${ }^{4}$ Enfermeira. Doutora em Enfermagem pelo Departamento de Enfermagem da UFC. Fortaleza (CE), Brasil. E-mail: priscilapetenf@gmail.com.

${ }^{5}$ Enfermeira. Doutora em Enfermagem pelo Departamento de Enfermagem da UFC. Fortaleza (CE), Brasil. E-mail: mlavinas@fortalnet.com.br.

${ }^{6}$ Enfermeira. Pós-doutora pela Universidade de São Paulo (USP). Ribeirão Preto (SP), Brasil. Professora-Associada do Departamento de Enfermagem da UFC. Fortaleza (CE), Brasil.E-mail: afcana@ufc.br.

Enderȩ̧o para correspondência: Camila Brasil Moreira. Rua Joaquim Nabuco, 3404 - São João do Tauape. Fortaleza (CE), Brasil. CEP: 60125-121.

E-mail: camilabrasil@alu.ufc.br.
} 


\section{INTRODUÇÃO}

No Brasil, o câncer de mama é o mais frequente nas mulheres, apresentando-se nas regiōes Sudeste (69/100 mil), Sul (65/100 mil), Centro-Oeste (48/100 mil) e Nordeste $(32 / 100 \mathrm{mil})$ como mais prevalente ${ }^{1}$. Esse quadro também se assemelha aos outros países onde, sem considerar os tumores da pele não melanoma, o câncer de mama é a neoplasia maligna mais comum entre as mulheres, respondendo por quase um em cada três casos de câncer diagnosticados entre o sexo feminino, e é a segunda principal causa de morte por câncer entre as mulheres ${ }^{2}$.

Estratégias de prevenção primária, secundária e terciária têm sido utilizadas com o objetivo de prevenir enfermidades, diagnosticá-las e tratá-las precocemente e minimizar seus efeitos na população, assegurando, a cada indivíduo, um padrão de vida adequado à manutenção da sua saúde ${ }^{3}$. Quando se fala em câncer de mama, a mamografia e o exame clínico das mamas são apontados como métodos eficazes na detecção precoce da doença ${ }^{4}$.

A mamografia convencional é o método de escolha para a detecção precoce nos programas de rastreamento do câncer de mama ${ }^{5}$, é um método de avaliação por imagem das lesões palpáveis e impalpáveis em mulheres com idade igual ou superior a 35 anos. Podendo ser essa complementada por outro exame, a ultrassonografia ${ }^{6}$. Ademais, salienta-se que o exame clínico das mamas deve ser realizado anualmente, para todas as mulheres com 40 anos ou mais. Sendo parte essencial da propedêutica para o diagnóstico de câncer da mama e devendo ser realizado como parte do exame físico e ginecológico, o exame clínico das mamas constitui-se como base para a solicitação dos exames complementares.

Entretanto, estudos comprovam que ainda existem lacunas no conhecimento das mulheres acerca de tais métodos o que gera uma reduzida adesão à prática desses exames $^{7-9}$. Essa realidade nos direciona para a importância da construçáo do conhecimento em saúde.

No Brasil, as críticas ao modelo tradicional de educação em saúde, oriundas das ciências biomédicas, vêm apontando uma visão simplista dos problemas de saúde pública ${ }^{10}$. O caráter muitas vezes superficial e/ou autoritário das açóes educativas em saúde tem incitado a busca de novas práticas e de uma problematização que contribuam para reconhecer as diversas vozes envolvidas na produçáo e no consumo de materiais educativos no campo da saúde pública ${ }^{11}$.

Estratégias de saúde mediadas pela tecnologia são utilizadas para modificar a forma como as relaçóes ocorrem em educação e saúde ${ }^{12-13}$. Essas estratégias permitem ao profissional utilizar materiais como o vídeo, pois é mais um instrumento para a construçáo de conhecimentos, desenvolvimento de habilidades e apoio à promoção da saúde. O uso desse recurso está em conformidade com a proposta educativa no processo pedagógico, com base em métodos de ensino colaborativo e interdisciplinar ${ }^{14}$.

Nessa perspectiva, buscando implementar açóes de educação em saúde, as tecnologias são processos concretos que, a partir de uma experiência cotidiana e da pesquisa, podem desenvolver um conjunto de atividades que serão produzidas e controladas pelos seres humanos, podendo ser veiculados como artefatos ou como saberes (estruturados). A tecnologia, então, contribui para a produção de conhecimentos a serem socializados, para dominar processos e produtos, a fim de transformar a utilização empírica em uma abordagem científica ${ }^{14}$.

$\mathrm{O}$ vídeo educativo já vem sendo utilizado desde a década de 1950, pois possibilita a exploração diferente dos temas abordados, bem como uma melhor visualizaçáo das informaçóes ${ }^{15}$. O vídeo pode despertar a curiosidade e o interesse pela investigação, bem como diversas outras competências, desde que utilizado de forma adequada e adaptada aos objetivos de aprendizagem ${ }^{16}$. Segundo Carvalho (1993), "a combinação de linguagens áudio e visual permite que a informação seja mais assimilada e, por isso, gera uma maior facilidade na aprendizagem" ${ }^{17}$. Portanto, pode-se referir que o vídeo educativo pode proporcionar ao indivíduo uma maior capacidade de reproduzir coerentemente o que foi compreendido das informaçóes oriundas dessa tecnologia educativa.

O preparo para o autocuidado e a promoção da saúde perpassa as meras informaçôes sobre como "prevenir" um agravo à saúde. Por isso, no que se refere à responsabilidade para a criação de açóes para o cuidado, a instauração de um processo de conhecimento faz-se necessária para o desenvolvimento de um trabalho educativo com pessoas envolvidas na busca pela qualidade de vida ${ }^{18}$.

Defronte ao contexto, destaca-se que o câncer de mama representa a primeira causa de morte, por câncer, entre as mulheres ${ }^{18}$, o que está relacionado ao diagnóstico em estádios avançados da doença.

Portanto, nota-se necessária a construção de uma tecnologia educacional para a realização de educação em saúde para a capacitação da comunidade acerca dos métodos diagnósticos do câncer de mama, a fim de tornar acessível o conhecimento sobre tais métodos de detecçáo da doença.

Diante do exposto, este estudo tem a finalidade de descrever a construção de um vídeo educativo sobre o câncer de mama em uma oficina virtual.

\section{MÉTODO}

Estudo do tipo desenvolvimento de tecnologia educativa que tem como objetivo descrever o desenvolvimento de recursos e serviços que contribuam para promover o conhecimento da sociedade acerca de um determinado tema ${ }^{19}$. 
Diante da prática assistencial como educador em saúde da temática câncer de mama nas unidades públicas de saúde e em instituiçóes educativas, percebeu-se a necessidade de um material educativo que possuísse um conteúdo coerente com a literatura científica e apresentasse aspectos que despertassem o interesse e proporcionasse a compreensão dos participantes do processo educativo acerca do câncer de mama. Essa inquietação estimulou a busca incessante por materiais que pudessem sanar essa necessidade percebida, entretanto não se obteve êxito, pois ainda não havia disponível um material que contemplasse os aspectos almejados, fato que incentivou a construção do material educativo relatado nesse estudo.

A construção ocorreu em julho de 2012, em quatro etapas: revisão narrativa da literatura, análise dos dados coletados, seleção dos dados pelos juízes e construção do material educativo.

Primeiramente, elaborou-se um roteiro com o conteúdo que seria abordado no vídeo; nessa etapa, foi realizada uma busca na literatura sobre estudos que abordassem o tema da oficina virtual, câncer de mama. Então, optou-se por realizar uma revisão narrativa da literatura que foi obtida a partir da busca de dados no site do Ministério da Saúde, do Instituto Nacional de Câncer José Alencar Gomes da Silva (INCA) e do Sismama, e da busca nas bases de dados PubMed e Lilacs. A busca foi realizada em julho de 2012 nas referidas bases de dados, e foram utilizados os descritores controlados "neoplasias da mama" (breast neoplasms), "detecção precoce do câncer" (early detection of cancer) e "métodos" (methods). Ao final da busca, obtiveram-se 82 artigos científicos. Consideraram-se, como critério de inclusão: estudos publicados nos últimos dez anos, que estivem disponíveis na íntegra, nos idiomas Português, Inglês e Espanhol, e que discursassem sobre os métodos de detecção do câncer de mama: exame clínico das mamas, autoexame das mamas, mamografia e/ou ultrassonografia. Entretanto, após aplicação dos critérios de inclusão e a leitura dos resumos desses estudos, foram selecionados apenas 15 artigos para análise.

$\mathrm{Na}$ análise dos dados, foram realizadas leituras criteriosas dos artigos na íntegra e, posteriormente, foram selecionados os dados relevantes para a construção do roteiro da oficina. Esses dados foram agrupados nas seguintes categorias: definição da doença, alterações mamárias sugestivas de câncer de mama, exame clínico das mamas, mamografia, ultrassonografia e autoexame das mamas.

$\mathrm{Na}$ terceira etapa, o roteiro foi avaliado por três juízes que foram escolhidos por atingirem aos seguintes critérios: conhecimento e prática na temática saúde da mulher e câncer de mama, e enfermeiro especialista em oncologia. Os três juízes escolhidos já haviam trabalhado com educação em saúde e desenvolvimento e/ou validação de material educativo sobre o tema. A entrega do roteiro primário se deu após a confirmaçáo do aceite do convite de participação da análise de conteúdo da oficina que foi enviada por e-mail para os três juízes selecionados. Os três juízes concordaram em participar dessa etapa do estudo e, no prazo de 30 dias, reenviaram o roteiro com as alteraçôes e/ou sugestóes. Ao receberem os três roteiros analisados, três autores do presente estudo se reuniram e, agregando as consideraçóes de cada juiz, construíram um roteiro final, conforme Quadro 1.

Após a obtenção dos dados que seriam abordados no vídeo educativo, a quarta etapa consistiu na gravação da oficina virtual, na qual se utilizaram os recursos filmadora digital, plano de fundo e figurino. A gravação se deu durante a realizaçáo de quatro oficinas e foi realizada no Laboratório de Comunicação em Enfermagem (LabCom) do Departamento de Enfermagem da Universidade Federal do Ceará. Posteriormente, contou-se com a avaliaçáo e participação de um especialista em mídias, editor de vídeos e imagem que realizou a ediçấo e confecção final da mídia digital com o recurso Windows Movie Maker 2007.

Conforme os preceitos ético-legais, a pesquisa atendeu às normas da resolução no $196 / 96$, além da aprovação do Comitê de Ética em Pesquisa do Hospital do Câncer do Ceará, no qual obteve a anuência com o protocolo de $\mathrm{n}^{\circ}$ 02/2006.

\section{RESULTADOS}

Obteve-se uma oficina virtual com duração de 12 minutos. A oficina é subdividida em quatro chamadas, conforme Quadro 2. A apresentaçáo do vídeo é realizada por uma enfermeira capacitada para sanar possíveis dúvidas dos participantes da sessão educativa posteriores à apresentação. Na primeira, aborda-se sobre o conceito de câncer de mama e conta-se com a apresentação de um vídeo extraído do YouTube que demonstra a divisão celular das células da mama e o desenvolvimento da doença. Na segunda chamada, são trazidos os dados epidemiológicos da doença no país com o auxílio de legendas durante a fala da apresentadora para favorecer a assimilação da informação. Na terceira chamada, faz-se uma explanação sobre as alteraçóes que podem ser observadas na mama sugestivas de câncer; são exibidas gravuras dessas alteraçôes à medida que se fala em cada uma delas, que são: vermelhidão ou hiperemia, presença de nódulos, secreçóes, abaulamentos, inchaço, retraçôes, coceira e presença de ferida. Na quarta chamada, citam-se os métodos de detecçáo da doença, com respectivas gravuras que ilustram como é realizado cada método, que foram referenciados conforme grau de importância e sensibilidade de detecção do câncer de mama, sendo eles: mamografia, exame clínico das mamas, ultrassom e 
Quadro 1. Conteúdo do roteiro final, segundo categorias de assunto

\begin{tabular}{|c|c|}
\hline Categorias & Conteúdo \\
\hline Definição da doença & $\begin{array}{l}\text { Olá telespectador! Hoje vamos conversar um pouco sobre o câncer de mama. Você sabe o que é câncer de mama? } 0 \text { câncer de } \\
\text { mama é uma doença maligna que surge na mama. (início do vídeo do YouTube) } 0 \text { seu aparecimento se dá devido à divisão } \\
\text { e multiplicação desordenada e acelerada das células da mama. Como vocês podem ver. Então elas vão se multiplicando, se } \\
\text { multiplicando e no final formam o que chamamos de tumor (Termina o vídeo do YouTube). }\end{array}$ \\
\hline Dados epidemiológicos & $\begin{array}{l}0 \text { câncer de mama é a principal causa de morte por câncer entre as mulheres. Em 2011, ocorreram mais de } 48.000 \text { casos novos da } \\
\text { doença só no Brasil e morreram aproximadamente } 11.864 \text { mulheres por essa doença. E a cada ano esses números aumentam. }\end{array}$ \\
\hline $\begin{array}{l}\text { Alterações mamárias } \\
\text { sugestivas de câncer de } \\
\text { mama }\end{array}$ & $\begin{array}{l}0 \text { câncer de mama geralmente não dói. Se esse tumor na mama estiver em estádios elevados, ou seja, quando faz certo tempo } \\
\text { que ele está se desenvolvendo ou até mesmo quando ele já está instalado, ele pode provocar o surgimento de alterações nessa } \\
\text { mama. Então, quando essas alterações surgem na mama de uma mulher, dizemos que elas são sugestivas de câncer de mama, } \\
\text { precisando, assim, que alguns exames sejam realizados para que essa mulher possa descobrir se é um câncer ou não. As alterações } \\
\text { são: (Cada figura referente à alteração aparece enquanto se fala) vermelhidão ou hiperemia, a pele da mama fica mais } \\
\text { avermelhada do que ela normalmente é e geralmente também está um pouco quente no local; edema ou inchaço, é normal as } \\
\text { mulheres possuírem uma mama um pouco maior que a outra, porém, quando uma mama é bem mais volumosa que a outra é } \\
\text { melhor procurar o serviço de saúde; nódulo na mama, se você visualizar ou palpar um caroço na sua mama, procure o serviço de } \\
\text { saúde; nódulo na axila, nossa mama é drenada por uma rede de vasos que podem levar para a axila alguma célula cancerosa que } \\
\text { estava na mama e lá na axila ela desenvolve outro tumor; abaulamento, ao avaliar sua mama, pode ser observado a presença de } \\
\text { uma elevação na pele da mama; retração do mamilo,o bico do seio fica virado para as laterais ou fica para dentro, ele não fica } \\
\text { protuso. É importante você saber que algumas mulheres já nascem com essa alteração e não é câncer; retração da pele, ao avaliar } \\
\text { a sua mama, pode ser visualizado um afundamento da pele na sua mama; coceira, geralmente ocorre na aréola ou ao redor do } \\
\text { mamilo. Presença de secreção, pode sair espontaneamente ou durante algum exame de avaliação das mamas uma secreção que } \\
\text { pode ter um dos três aspectos: avermelhada, cor de coca-cola ou esbranquiçada e mal cheirosa. Pode ser também observado a } \\
\text { presença de uma ferida na mama, mas geralmente essa alteração só acontece quando a doença já está bem avançada. }\end{array}$ \\
\hline $\begin{array}{l}\text { Exame clínico das } \\
\text { mamas, mamografia, } \\
\text { ultrassonografia e } \\
\text { autoexame das mamas }\end{array}$ & 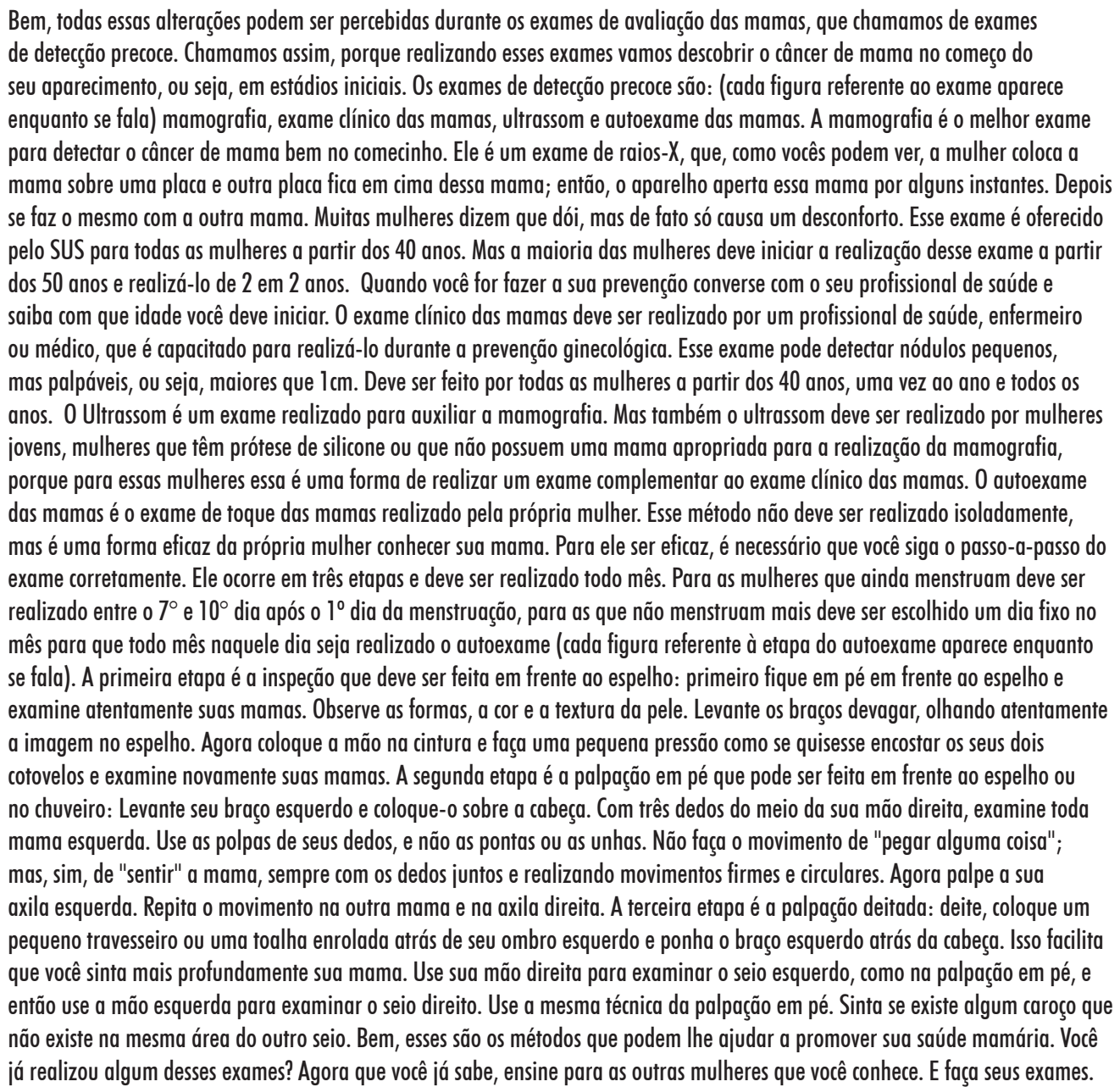 \\
\hline
\end{tabular}


Quadro 2. Divisão das chamadas do vídeo educativo, segundo categorias de assunto abordado

\begin{tabular}{|l|l|}
\hline \multicolumn{1}{|c|}{ Secção vídeo } & \multicolumn{1}{c|}{ Categorias } \\
\hline Chamada 1 & Definição da doença \\
\hline Chamada 2 & Dados epidemiológicos \\
\hline Chamada 3 & $\begin{array}{l}\text { Alterações mamárias sugestivas } \\
\text { de câncer de mama }\end{array}$ \\
\hline Chamada 4 & $\begin{array}{l}\text { Exame clínico das mamas, } \\
\text { mamografia, ultrassonografia e } \\
\text { autoexame das mamas }\end{array}$ \\
\hline
\end{tabular}

autoexame das mamas. Ao final, faz-se um apelo para que a mulher se sinta motivada para a busca pelos serviços de saúde com o intuito de promover sua saúde mamária e sinta-se estimulada para a prática dos métodos de detecção do câncer de mama.

\section{DISCUSSÃO}

Nos últimos anos, tem se discutido o desenvolvimento de ações abrangentes para o controle do câncer, nos diferentes níveis de atuação, destacando: a promoção da saúde, a detecção precoce, a assistência aos pacientes, a comunicação e a mobilizaçáo social, e como são fundamentais.

A literatura consultada refere que até o momento não se identificou nenhuma população feminina isenta do risco de desenvolver o câncer mamário, e esse fato deve ser considerado em programas de promoção da saúde?. Os métodos de detecção precoce dessa doença não podem ser excluídos da atenção da equipe de saúde pública, principalmente quando se refere à informação. Toda ação voltada para a construçáo do conhecimento acerca dessa temática deve ser divulgada aos profissionais da saúde com o intuito de estimulá-los e alertá-los da importância em informar a comunidade acerca da detecçâo precoce do câncer de mama.

A informação como estratégia a ser utilizada para a prevenção deve ser trabalhada juntamente com a questáo da autonomia do indivíduo e compreensão de que a educaçáo em saúde é uma construçáo do indivíduo dentro de um contexto sócio cultural e individual.

A elaboração de materiais educativos, sobre orientaçôes às mulheres acerca do câncer de mama, pode incentivar a construçáo de outros materiais didáticos, especialmente na área da enfermagem, pois ela precisa ampliar o exercício contínuo da atitude crítica diante das próprias produçôes; observar além do que lhe apresenta, como mais uma forma de obter maior visibilidade sobre o que a cerca; registrar e divulgar a produçáo do seu conhecimento e estar pronta a enfrentar os desafios impostos pelas ciências na profissão ${ }^{20}$.
No contexto da educação em saúde, a contribuição de tecnologias educativas e o papel desse recurso sáo voltados para promoção da saúde, prevenção de complicaçóes, desenvolvimento de habilidades e favorecimento da autonomia e confiança do paciente. Como um componente da equipe interdisciplinar que desempenha a funçáo de educador, o enfermeiro deve participar do processo de criação, desenvolvimento e avaliação do material educativo ${ }^{21}$.

A detecção precoce de uma doença é possível por meio de educação para o diagnóstico precoce em pessoas sintomáticas ou do rastreamento (triagem) em populaçóes assintomáticas. É essencial educar a população e os profissionais de saúde para o reconhecimento dos sinais e sintomas precoces do câncer, contribuindo para sua detecção em estágios menos avançados e aumentando as chances de sucesso do tratamento preconizado ${ }^{22}$.

\section{CONCLUSÃO}

Compete ao profissional da saúde a responsabilidade de promover a saúde da comunidade, portanto, faz-se necessário o desenvolvimento de avanços tecnológicos que assegurem o compromisso social, a luta e o combate às doenças, como por exemplo, através da elaboração de materiais que auxiliem a educação em saúde.

No âmbito da saúde coletiva, existe uma grande preocupação em relação às doenças crônicas, uma vez que muitas dessas são desconhecidas pela populaçáo que não se torna adepta aos hábitos de vida saudáveis e aos métodos de detecçáo dessas doenças, ficando assim mais vulneráveis a adquiri-las. No caso do câncer de mama, fala-se em prevenção secundária através dos métodos de detecção precoce da doença.

Sendo assim, considera-se de extrema importância a construção do conhecimento acerca desses métodos, sendo papel do enfermeiro como educador em saúde fornecer subsídios para que as informaçôes acerca dessa doença sejam fornecidas à comunidade. A transmissão do conhecimento deve ser realizada de forma a favorecer a compreensão da informação transmitida, a fim de que o processo de assimilação do conteúdo e a construção do saber sejam atingidos efetivamente.

O desenvolvimento da oficina virtual como uma tecnologia educativa pode facilitar a construçáo do conhecimento por se se tratar de um material educativo com recursos visuais e auditivos, com transmissão de livre acesso em qualquer lugar via internet.

\section{CONTRIBUIÇÕES}

Camila Brasil Moreira e Elizian Braga Rodrigues Bernardo contribuíram na concepção e planejamento do projeto de pesquisa. Priscila de Souza Aquino e Hellen 
Lívia Oliveira Catunda contribuíram na obtenção e/ou análise e interpretação dos dados. Míria Conceição Lavinas Santos e Ana Fátima Carvalho Fernandes contribuíram na redação e revisão crítica.

\section{Declaraçáo de Conflito de Interesses: Nada a Declarar.}

\section{REFÊRENCIAS}

1. Ministério da Saúde (Brasil). Sismama. Taxas de incidência de neoplasia maligna da mama estimadas para o ano de 2012 [Internet]. 2012 [acesso 2013 Mai 22]. Disponível em: http://tabnet.datasus.gov.br/cgi/tabcgi. exe?siscolo/sismama/DEF/CEMMAMA4.def

2. American Cancer Society. Breast cancer facts \& figures 2011-2012. Atlanta: American Cancer Society, Inc; 2011.

3. Ricci MD, Pinotti M, Pinotti JA. Perspectivas da ultrasonografia na detecção e diagnóstico do câncer de mama. Rev ginecol obstet. 2000; 11(3): 156-60.

4. Santos GD, Chubaci RYS. O conhecimento sobre o câncer de mama e a mamografia das mulheres idosas frequentadoras de centros de convivência em São Paulo (SP, Brasil). Ciênc. saúde coletiva. 2011; 16(5): 2533-40.

5. Francioni FF, Coelho MS. A superação do déficit de conhecimento no convívio com uma condição crônica de saúde: a percepção da necessidade da ação educativa. Texto contexto Enferm. 2004; 13(1): 156-62.

6. Instituto Nacional de Câncer (Brasil). Controle do câncer de mama: documento de consenso. Rio de Janeiro: INCA; 2004.

7. Ferreira MLM, Oliveira C. Conhecimento e significado para funcionárias de indústrias têxteis sobre prevenção do câncer do colo-uterino e detecção precoce do câncer da mama. Rev bras cancerol. 2006; 52(1): 5-15.

8. Batiston AP, Tamaki EMT, Santos MLM, Cazola LHO. Método de deteç̧ão do câncer de mama e suas implicações. Cogitare enferm. 2009; 14(1): 59-64.

9. Fogaça EIC, Garrote LF. Câncer de mama: atenção primária e detecção precoce. Arq ciênc saúde. 2004; 11(3): 179-81.

10. Gómez GO. Comunicação, educação e novas tecnologias: tríade do século XXI. Comunicação \& Educação 2002; 8(23):57-70.
11. Monteiro S, Vargas E. Educação, comunicação e tecnologia educacional: interfaces com o campo da saúde. Rio de Janeiro: Editora Fiocruz; 2006.

12. Brasil. Lei no 9394, de 20 dez 1996. Estabelece diretrizes e bases da educação nacional. Diário Oficial [da] Republica Federativa do Brasil n0 248. Brasília, DF, 1996 dez 23; Seção 1, fls.27833-41.

13. Conselho Nacional de Educação (Brasil). Resolução CNE/CES No 3, de 7 de novembro de 2001. Institui diretrizes curriculares nacionais para o curso de graduação em enfermagem. Diário Oficial [da] Republica Federativa do Brasil. Brasília, DF, 2001 Nov 8; Seção1, fl. 37.

14. Aguiar RV, Cassiani SHB. Desenvolvimento e avaliação de ambiente virtual de aprendizagem em curso profissionalizante de enfermagem. Rev latinoam enferm. 2007; 15(6):1086-91.

15. Williams S. Education and the information revolution. In: Paine N, editor. Open learning in transition. London: Kogan Page; 1988. p.3-13.

16. Coutinho CP. A influência das teorias cognitivas na investigação em Tecnologia Educativa: pressupostos teóricos e metodológicos, expectativas e resultados. Rev Port Educa. 2008; 21 (1): 101-27.

17. Carvalho AAAS. Utilização e exploração de documentos audiovisuais. Rev Port Educação. 1993; 6(3): 113-21.

18. Monteiro S, Vargas E, Cruz M. Desenvolvimento e uso de tecnologias educacionais no contexto da aids e da saúde reprodutiva: reflexōes e perspectivas. In: Monteiro S, Vargas $\mathrm{E}$, organizadores. Educação, comunicação e tecnologia educacional. Rio de Janeiro: Editora Fiocruz; 2006. p.27-34.

19. Polit DF, Hungler BP. Fundamentos de pesquisa em enfermagem. 3. ed. Porto Alegre: Artes Médicas; 1995.

20. Oliveira MS, Fernandes AFC, Sawada NO. Manual educativo para o autocuidado da mulher mastectomizada: um estudo de validação. Texto \& contexto enferm (online) [Internet]. 2008 [acesso 2012 Nov 19]; 17(1):115-23. Disponível em: http://www.scielo.br/pdf/ tce/v17n1/13.pdf

21. Moreira MF, Silva MIT. Readability of the educational material written for diabetic patients. Online braz $\mathrm{j}$ nurs (online) [Internet]. 2005 [acesso 2012 Nov 19]; 4(2). Disponível em: http://www.nepae.uff.br//siteantigo/ objn 402moreiraetal.htm.

22. Thuler LC. Considerações sobre a prevenção do câncer de mama feminino. Rev bras cancerol. 2003; 49(4): 227-38. 


\begin{abstract}
Introduction: Health education activities encourage diseases prevention, health promotion and engagement of the population in matters related to health and quality of life through knowledge construction. Objective: To describe the elaboration of an educational video about breast cancer in a virtual workshop. Method: Study of the type of development of of educational technology. The construction of the educational material occurred in June 2012, and had four steps: narrative review of the literature, analysis of data collected, data selection and construction of the material. Results: It was obtained an educational video which addresses what is breast cancer, the changes observed in the breast that could suggest cancer and methods for its detection: mammography, clinical breast examination, breast ultrasound and breast self-examination. Conclusion: Nurses as health professionals are responsible for promoting the health of the community, thus they should develop advances in nursing in order to ensure the social commitment, besides to struggle and fight against diseases using, for example, preparation of materials to assist health education. The virtual workshop as an educational technology can facilitate the construction of knowledge so as to promote health. Key words: Breast Neoplasms-prevention \& control; Early Diagnosis; Audiovisual Aids; Educational Technology; Health Communication
\end{abstract}

\title{
Resumen
}

Introducción: Las actividades de educación en la salud fomentan la prevención de enfermedades, la promoción de la salud y el comprometimiento de la población en los temas relacionados a la salud y la calidad de vida a través de la construcción del conocimiento. Objetivo: Describir la construcción de un video educativo sobre el cáncer de mama en un taller virtual. Método: Estudio del tipo desarrollo de la tecnología educativa. La construcción del material educativo ocurrió en junio de 2012, en cuatro etapas: revisión narrativa de literatura, análisis de los datos recogidos, selección de los datos y construcción del material. Resultados: Se obtuvo un video educativo sobre la definición del cáncer de la mama, las alteraciones físicas en la mama, que sugiere la presencia del cáncer y los métodos de detección de la enfermedad: mamografía, prueba clínica de las mamas, ultrasonido de mama y el autoexamen de las mamas. Conclusión: La enfermera como profesional de la salud es responsable por promocionar la salud en la comunidad, debiendo desarrollar los avances en la enfermería que garanticen el compromiso social, la lucha y el combate a las enfermedades, como la preparación de materiales que auxilien a la educación en salud. El taller virtual como una tecnología educativa puede facilitar la construcción del conocimiento de manera a promocionar salud.

Palabras clave: Neoplasias de la Mama-prevencíon \& control; Diagnóstico Precoz; Medios Audiovisuales; Tecnología Educacional; Comunicacíón em Salud 